FORMATION Formation emploi

Revue française de sciences sociales

156 | Octobre-Décembre

Les apories de la professionnalisation des formations initiales

Présentation de l'ouvrage d'Isabelle Harlé, Penser le curriculum scolaire : le regard croisé de la sociologie, des didactiques et de l'histoire

Aymeric Le Corre

CpenEdition

Journals

Édition électronique

URL : https://journals.openedition.org/formationemploi/10269

DOI : 10.4000/formationemploi.10269

ISSN : 2107-0946

Éditeur

La Documentation française

Édition imprimée

Date de publication : 31 décembre 2021

Pagination : 157-160

ISSN : 0759-6340

Référence électronique

Aymeric Le Corre, «Présentation de l'ouvrage d'Isabelle Harlé, Penser le curriculum scolaire : le regard croisé de la sociologie, des didactiques et de l'histoire », Formation emploi [En ligne], 156 | Octobre-

Décembre, mis en ligne le 31 décembre 2021, consulté le 03 mars 2022. URL : http://

journals.openedition.org/formationemploi/10269; DOI : https://doi.org/10.4000/formationemploi. 10269

(c) Tous droits réservés 


\title{
Note de Lecture
}

\section{Présentation de l'ouvrage d'Isabelle Harlé, Penser le curriculum scolaire: le regard croisé de la sociologie, des didactiques et de l'histoire}

\author{
Par Aymeric Le Corre \\ Doctorant en Sociologie, Université de Poitiers - Groupe de REcherches Sociologiques sur les \\ sociétés COntemporaines (GRESCO)
}

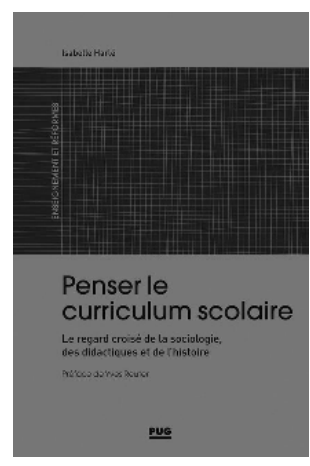

Comme l'indique le sous-titre de cet ouvrage, Isabelle Harlé invite le lecteur souhaitant se saisir des contenus d'enseignement à aborder cette tâche à l'aune de la sociologie, des didactiques et de l'histoire. Dans ce livre tiré de son habilitation à diriger des recherches, l'auteure se saisit de ses nombreux travaux scientifiques questionnant les contenus scolaires et qui ont jalonné son parcours de chercheuse, mais aussi d'enseignante.

Selon Isabelle Harlé, la sociologie du curriculum, dont les travaux traitent de la question des choix et de l'organisation des contenus dans le champ scolaire, est encore aujourd'hui à la marge de la sociologie de l'éducation. En livrant quelques ficelles tirées de l'expérience de son auteure, cet ouvrage agit comme un médiateur de ce courant britannique, ce qui est d'autant plus utile que les concepts développés peuvent parfois être perçus comme complexes à manier. Tout au long de l'ouvrage, l'attention portée par l'auteure au travail d'écriture amène le lecteur à prendre conscience de la construction sociale des savoirs et permet, par les nombreuses questions relevées par l'auteure, de nous guider dans la réflexion.

Le premier chapitre de l'ouvrage aborde la manière dont les contenus d'enseignement sont traités par deux disciplines, la didactique et la sociologie. Partant des débats des années 1990 qui interrogent la quasi-absence de la sociologie dans l'analyse des savoirs scolaires, l'auteure se saisit du cadre théorique offert par la sociologie du curriculum pour ramener la discipline au centre de l'analyse des contenus. Pour cela, elle s'appuie sur ses précédents travaux pour montrer la richesse de ce courant sociologique britannique et américain (Forquin, 1997) dans le processus de dénaturalisation des savoirs scolaires. Prolongeant la médiatisation de ce courant sociologique initiée par JeanClaude Forquin (2008), elle s'applique à montrer comment cette sociologie amène à 
questionner les modes de sélection et de diffusion des savoirs en fonction des publics scolaires. Il s'agit aussi pour l'auteure d'indiquer les différences dans les approches didactique et sociologique concernant l'analyse des savoirs, tout en montrant qu'une collaboration disciplinaire est possible.

Le second chapitre est consacré aux dialogues entre didactique et sociologie à travers la médiation de l'Histoire. Par la mobilisation de travaux historiques de l'éducation et les " moments-clés " (p. 68) qu'ils révèlent, l'auteure dénaturalise le savoir scolaire et montre que le dialogue entre sociologie et didactique n'est possible qu'à travers une analyse des enjeux sociaux autour des savoirs. Progressivement, et par l'emploi de références disciplinaires variées, elle amène le lecteur à relativiser des notions habituellement saisies par les enseignants comme allant de soi, comme les disciplines et les contenus, et élargit l'analyse curriculaire aux savoirs, aux savoir-faire, aux techniques et valeurs que ces notions traversent. Enfin, à travers le concept de «configuration disciplinaire » (p. 81), emprunté au didacticien Yves Reuter, elle réalise une synthèse des trois disciplines qu'elle articule dans son usage de la sociologie du curriculum.

Le troisième chapitre poursuit l'implication des trois disciplines autour du concept de configuration que l'auteure définit dans le chapitre précédent. Ici, il s'agit pour elle d'apporter la démonstration que ce concept, issu de la didactique, peut être mobilisé par les sociologues à travers l'ajout de concepts sociologiques comme la forme scolaire (Vincent, 1994) ou encore la stratification (Young, 1997). Pour ce faire, l'auteure s'appuie sur des exemples tirés de ses recherches, mais aussi d'ateliers menés dans les Instituts nationaux supérieurs du professorat et de l'éducation auprès des masters Métiers de l'enseignement, de l'éducation et de la formation. Ses expériences de chercheuse, mais aussi d'enseignante, illustrent parfaitement la démarche d'appropriation du concept par une discipline comme la sociologie. L'ensemble participe clairement à la démarche de dénaturalisation des curricula et à l'appropriation des questions que cette relativisation suppose.

Pour finir, le quatrième chapitre aborde les questionnements qui peuvent se poursuivre pour penser les réformes curriculaires dans les différents espaces scolaires ou non. Pour ce faire, l'auteure encourage le chercheur à se saisir d'une démarche sociohistorique couplée au concept de forme scolaire (Vincent, 1994) permettant d'analyser les contenus et plus largement la structure des curricula. L'époque actuelle, sous l'égide de la lutte contre les inégalités et d'une politique volontariste de réduction de l'échec scolaire, semble propice au développement de dispositifs hors disciplines et donc à une moindre place accordée aux savoirs scolaires. L'auteure interroge ce changement dans l'école et pose à juste titre la question suivante : "Est-il prouvé que supprimer les frontières entre savoirs scolaires et savoirs de la vie courante permettrait à tous les élèves de réussir?» (p. 128). 
Cet ouvrage est certainement des plus stimulants pour des chercheurs ou apprentis chercheurs en quête de moyens leur permettant de se saisir des savoirs scolaires. Il apporte notamment deux éléments importants :

Premièrement, il permet de prolonger le débat entamé il y a de cela plusieurs années entre les sociologues et les didacticiens sur la question des "savoirs scolaires ». L'auteure s'est saisie de concepts mêlant ces deux disciplines pour faire la synthèse de ses travaux, mais aussi offrir des perspectives de recherche aux jeunes chercheurs et, pourquoi pas, donner naissance à de nouvelles collaborations disciplinaires. Elle a su montrer que le dialogue entre la didactique et la sociologie est bien possible, même si, elle l'avoue, celui-ci s'inscrit sous certaines conditions.

Le second apport de cet ouvrage est de remettre en avant ce qu'avait réalisé JeanClaude Forquin par le passé, c'est-à-dire de mettre au cœur de l'analyse la sociologie du curriculum. En s'appropriant les travaux des sociologues britanniques et américains et en les rendant accessibles par l'apport d'exemples empiriques tirés de sa pratique d'enseignante-chercheuse, l'auteure transforme un objet opaque, la sociologie du curriculum, en notion concrète. $S$ 'il reste du chemin à parcourir pour placer ce courant un peu plus au centre des travaux de la sociologie de l'éducation, il est certain que cet ouvrage peut permettre de donner naissance à de nouveaux questionnements et à des champs de recherche plus développés.

La seule critique qui peut être adressée à cet ouvrage concerne la structuration des chapitres, qui peut paraître déséquilibrée et laisse le lecteur sur sa faim tant il aimerait poursuivre la réflexion avec l'auteure.

Une chose est sûre, ce livre, dont la question transversale portait sur ce qu'est un sociologue du curriculum au sein des sciences de l'éducation et dans la formation en INSPE, répond que celui-ci s'inscrit comme un médiateur entre la discipline sociologique et son lecteur.

\section{- Référence de l'ouvrage}

Isabelle Harlé (2021), Penser le curriculum scolaire : le regard croisé de la sociologie, des didactiques et de l'histoire, Grenoble, PUG, Collection «Enseignement et réformes », mars.

\section{- Bibliographie}

Forquin J.-C. (1997), Les sociologues de l'éducation américains et britanniques : présentation et choix de textes, Paris, De Boeck université, INRP.

Forquin J.-CL. (2008), Sociologie du curriculum, Rennes, PUR, collection « Paideia ». 
Vincent G. (1994), Groupe de recherches sur le procès de socialisation (Lyon, France), et Association internationale des sociologues de langue française (éd.), L'éducation prisonnière de la forme scolaire? Scolarisation et socialisation dans les sociétés industrielles, Lyon, Presses universitaires de Lyon.

Young M. (1997), « Les programmes scolaires considérés du point de vue de la sociologie de la connaissance " in Forquin J.-C, Les sociologues de l'éducation américains et britanniques : présentation et choix de textes, Paris, De Boeck université, INRP, pp. 173-199. 